\title{
Parametric study of femtosecond inscription of microstructures for OCT artefact fabrication
}

\author{
Janarthanan Rasakanthan ${ }^{1 \mathrm{a}}$, Graham Chun Bon Lee ${ }^{\mathrm{a}}$, Peter. D. Woolliams ${ }^{\mathrm{b}}$, \\ Kate Sugden ${ }^{\mathrm{a}}$

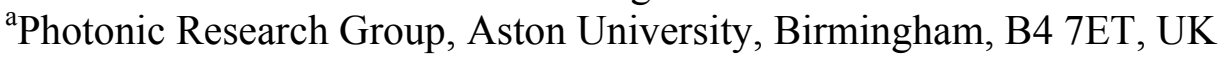 \\ ${ }^{\mathrm{b}}$ Biophotonics Group, National Physical Laboratory, Hampton Road, Middlesex, \\ TW11 0LW, UK
}

\begin{abstract}
As optical coherence tomography (OCT) becomes widespread, validation and characterization of systems becomes important. Reference standards are required to qualitatively and quantitatively measure the performance between difference systems. This would allow the performance degradation of the system over time to be monitored. In this report, the properties of the femtosecond inscribed structures from three different systems for making suitable OCT characterization artefacts (phantoms) are analyzed. The parameter test samples are directly inscribed inside transparent materials. The structures are characterized using an optical microscope and a swept-source OCT. The high reproducibility of the inscribed structures shows high potential for producing multi-modality OCT calibration and characterization phantoms. Such that a single artefact can be used to characterize multiple performance parameters such the resolution, linearity, distortion, and imaging depths.
\end{abstract}

KEYWORDS: optical coherence tomography, standardization, phantoms, calibration, characterization, femtosecond inscription

\section{INTRODUCTION}

Optical coherence tomography (OCT) is a non-invasive, three-dimensional imaging methodology ${ }^{1,2}$. As the use of optical coherence tomography system becomes more widespread, verifying the performance against a reference standard becomes vital. A reference standard is required to measure the performance of difference systems as well as measure the performance degradation of the system over time. Therefore reliable measurement artefacts (phantoms) and processing techniques are essential to quantify the system performance, as well to have a qualitative control.

Standard artefacts are used for validating and optimizing the performance of the system by the vendors and for routine quality control checks at the clinics. The main advantage of using standard phantoms is intra-laboratory consistency of the reported system's performance.

Regulatory bodies such as European Council require the validation of the imaging system's performance using standard phantoms to ensure the uniformity of the reported performance between institutions and over time $e^{3}$. Lack of standard calibration and characterization using known, wellcalibrated phantoms leads to the uncertainties in the reported results. Therefore, validating the performance of the OCT using a globally accepted standard would increase confidence in the reported performance of the systems but requires easy to use, reproducible phantoms. Ideally, multiple performance matrices should be characterized using a single phantom or set of data.

In this work, we investigate the properties of the femtosecond laser inscribed structures by three different laser systems and the suitability of each system for the production of OCT characterization artefacts. In order to do this, the parameter space test samples were inscribed inside the bulk of

${ }^{1}$ rasakanj@aston.ac.uk

Optical Coherence Tomography and Coherence Domain Optical Methods in Biomedicine XVI, edited by Joseph A. Izatt, James G. Fujimoto, Valery V. Tuchin, Proc. of SPIE Vol. 8213, 82133N (C) 2012 SPIE - CCC code: 1605-7422/12/\$18 - doi: 10.1117/12.907053 
transparent materials (fused silica, borosilicate, doped-BK7 and PMMA). The properties of the inscribed structures were characterized using an optical microscope and a swept source OCT system.

Important parameters of the OCT systems include resolution, sensitivity, contrast, distortions, linearity and imaging depth. The three-dimensional point spread function (PSF) of the system can be estimated by measuring an infinitesimally small structure (i.e. structures much smaller than the resolution of an OCT $)^{4-6}$. The sensitivity of the OCT system can be measured using microstructures with varying refractive index. The distortion of the system can be measured by imaging artefacts with parallel lines or grids ${ }^{7}$.

Experimental measurement of PSF has been demonstrated by Agrawal et al and Woolliams et al who used particles suspended in aqua solution and embedded in a matrix to measure the three dimensional point spread function of the OCT system ${ }^{4,5}$. With suitable artefacts it is also possible to measure the contrast and modulation transfer function (MTF) of the OCT system ${ }^{8}$. However there are limitations and difficulties associated with the particle based artefacts and in 2011 Tomlins et al showed the possibility of using femtosecond inscribed microstructures as PFS artefacts ${ }^{6}$.

Femtosecond laser inscription can be used to produce potential artefacts that can assess all these performances matrices. Since the material modifications are due to non-linear absorption, it is only around the focal point of the femtosecond laser pulse that the absorbed energy density exceeds the critical one required to modify the material ${ }^{9-15}$. Sample translation or beam scanning, enables complex three-dimensional structures to be fabricated within the bulk of transparent media in a simple, single stage procedure.

For this work, a series of lines were written at different depths within the sample, shown schematically in Figure 1. The change in induced refractive index is related to the inscription energy. However, other inscription parameters affect the quality of the lines. To explore this, the pulse energy, speed of translation and depth of the feature were varied since these are the main parameters that affect the quality of the inscribed structure. In addition, suitability of different materials (fused silica, doped BK7, Borosilicate and PMMA) for making OCT phantoms were investigated. The samples were then characterized using an optical microscope equipped with quantitative phase microscopy (QPM) and a swept source OCT system.

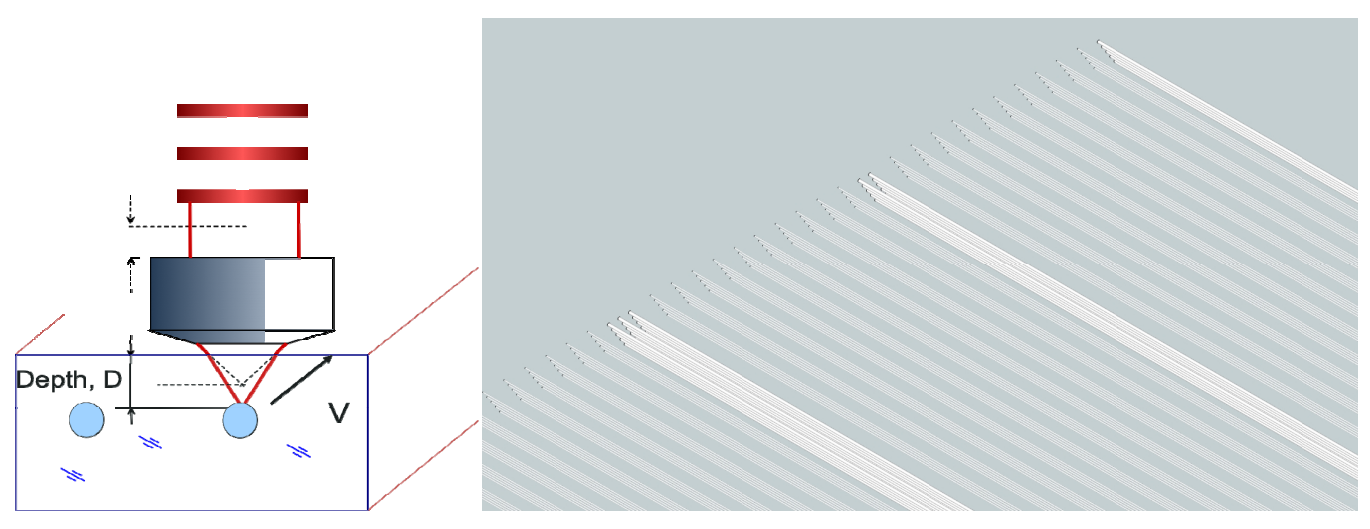

Fig. 1: a) The schematics of the inscription process. b) 3D schematics of the artifact.

\section{METHODS}

\subsection{Fabrication of the artefacts}


In this study, the parameter space test samples were inscribed by three different laser systems. Two of the systems are Ti:Sapphire lasers and both operate at a central wavelength of $800 \mathrm{~nm}$. The repetition rates are $11 \mathrm{MHz}$ (High-Rep800) and $1 \mathrm{kHz}$ (Low-Rep800) and the pulse durations 50 and $110 \mathrm{fs}$ respectively. The third system is Nd:YAG s-pulse HP laser (Low-Rep1030), which operates at a center wavelength of $1026 \mathrm{~nm}$ with $100 \mathrm{kHz}$ repetition rate and has $\sim 500 \mathrm{fs}$ pulse duration. Figure 2 illustrates the simplified layout of the experimental setup of Low-Rep800 system.

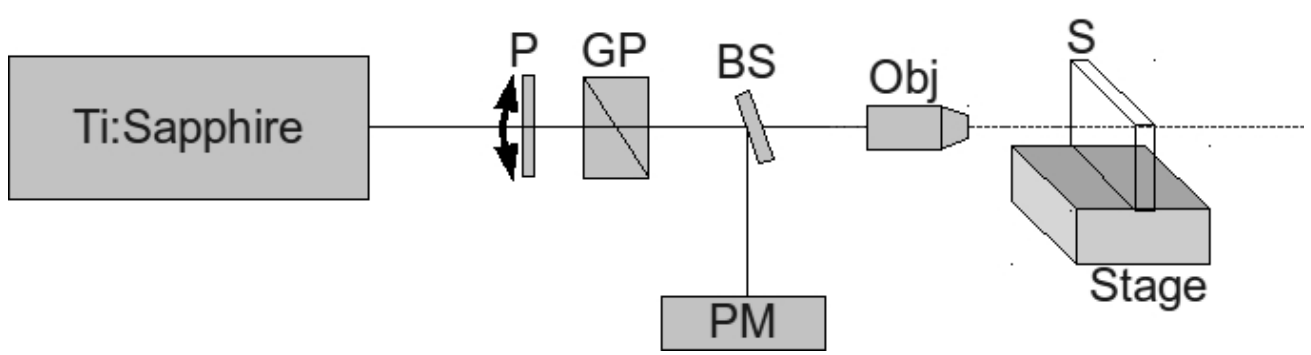

Figure 2: Schematics of the experimental setup of Low-Rep800 system. Where P is the half wave plate, GP is the Glan-prism, BS is the beam splitter, PM is the power meter, $\mathrm{S}$ is the sample mounted on the translation stage.

The pulse energy of all three systems can be adjusted using a half-wave plate and a Glan-prism (labeled as P and GP in Figure 2). The half wave plate rotates the linearly polarized wave, and Glanprism only transmits the vertically polarized component of the beam. Therefore, rotating the half-wave plate without altering the polarization of the beam varies the energy of the laser beam.

The samples are mounted on computer controlled multi-dimension air-bearing translation stage and translated perpendicular to the laser beam at constant velocities, relative to the beam waist to fabricate the intended structure.

During the inscription process, structures at the lowest level were fabricated first in order to avoid scattering by modified regions as illustrated in Figure 3.

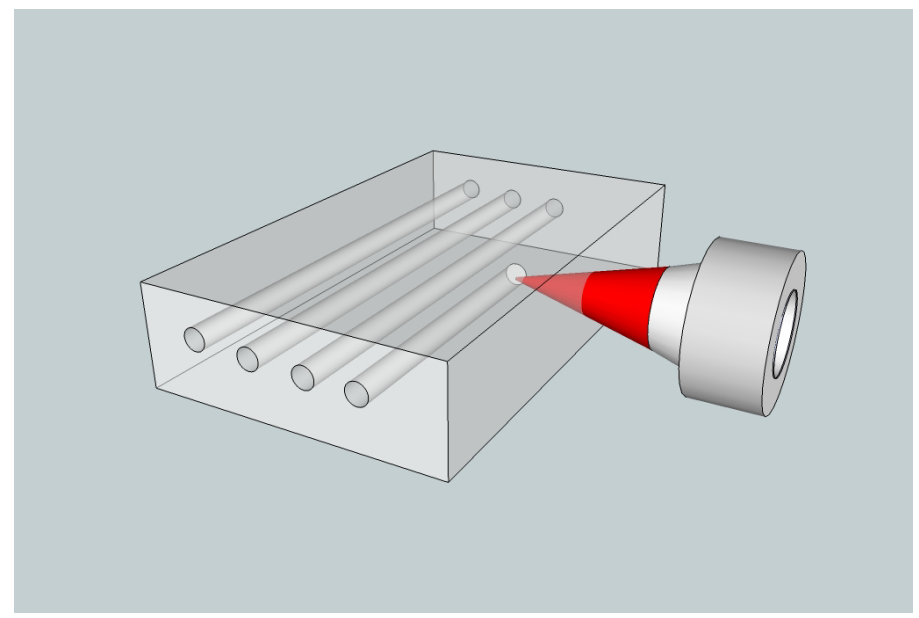

Figure 3: schematics representation of writing multiple lines.

The main variables that influence the inscribed structures are fluence, repetition rate, wavelength, speed and depth of inscription. Table 2 shows parameter ranges used in the samples presented in this study. Due to the nature of the low repetition laser, the inscribed structures are elliptical in the axial direction ${ }^{9}$. The magnitude of the elongation increases with increasing energy. These elongations can 
be minimized by either using a cylindrical lens or slits ${ }^{9}$. This is not an issue in the high-repetition system, where thermal accumulation melts the material to produce symmetrical structures ${ }^{9}$.

Table 2: Summary of the parameter ranges investigated.

\begin{tabular}{|l|l|l|}
\hline & Low-Rep 800 & Low-Rep1026 \\
\hline Energy & $16.89-80 \mu \mathrm{W}$ & $12.53-155.5 \mathrm{~mW}$ \\
\hline Speed & $0.1-2 \mathrm{~mm} / \mathrm{s}$ & $0.1-15 \mathrm{~mm} / \mathrm{s}$ \\
\hline Depth & $100-775 \mathrm{um}$ & $100-625 \mathrm{um}$ \\
\hline
\end{tabular}

Figure 4 show a schematic of the parameter space sample design. The translation speed varies along the $\mathrm{x}$-axis (i.e. each column is written at different speed), the inscription energy varies along y-axis, and depth of inscription varies along the z-axis. The size of the structures is limited by either the size of the sample, stage motion and/or depth of focus.
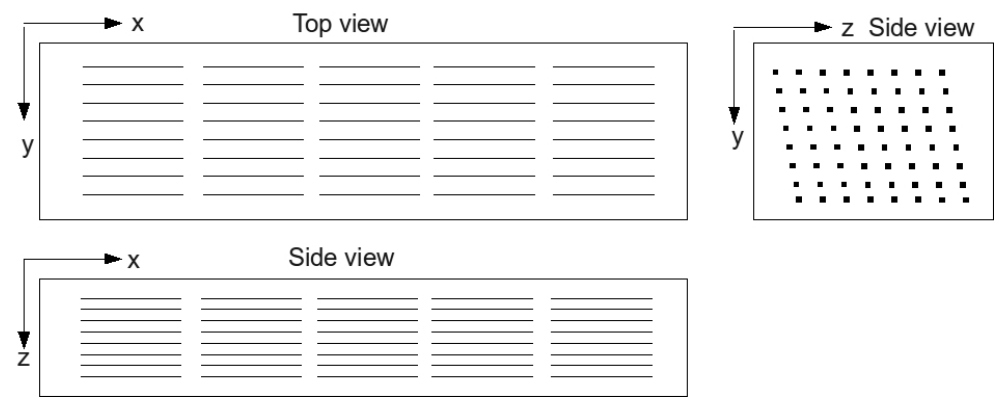

Figure 4: Schematics of the artefacts

The layers were offset so that multiple scattering effects are avoided during OCT measurement. The lines were written in pairs where both lines in the pair were inscribed with same energy, speed and depth to verify the reproducibility of the inscribed structures. Figure 5 shows the schematic of a line pair inscribed at different depths but with the same energy.

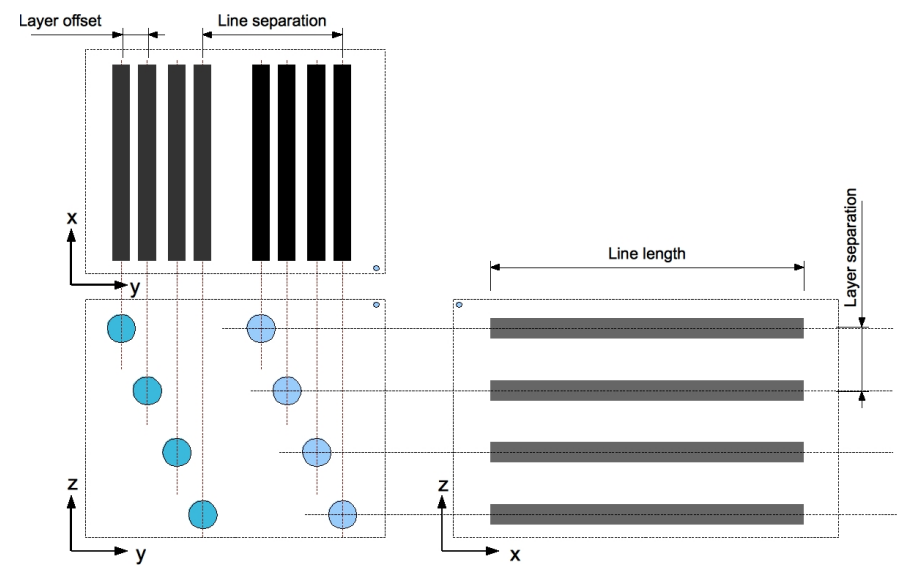

Figure 5: Schematics of a line pair at different depth

In addition, artefacts were repeated few times to ensure the reproducibility. The lines pairs are separated into sets using index lines helps to identify the lines during the characterization as shown in Figure 6. 


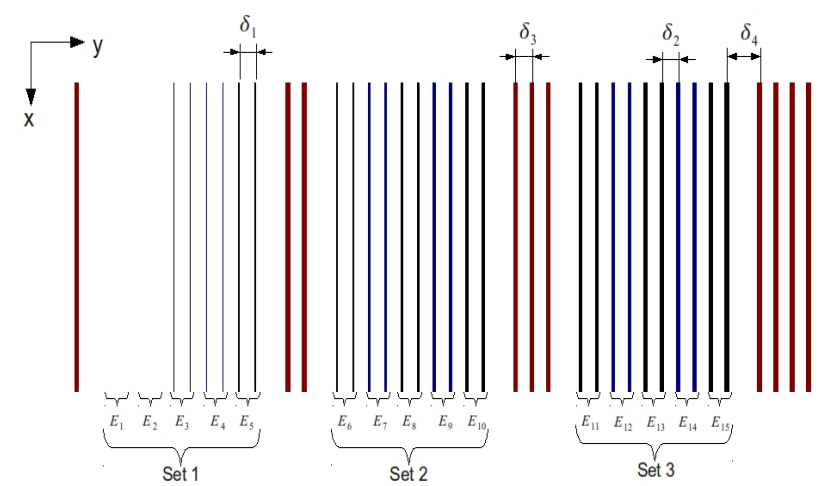

Figure 6: Illustrate the lines pairs with increasing energy, separated by index-lines (red).

\subsection{Characterization of the artefacts}

The properties of the inscribed structures can be characterized using a multitude of techniques such as refractive near field profiling and quantitative phase microcopy $(\mathrm{QPM})^{16-18}$. In this study, structures are characterized using an optical microscope (Axioscope-2 MOT plus, Zeiss) equipped with QPM and a swept source OCT operating at the center wavelength of $1305 \mathrm{~nm}$.

\section{RESULTS AND DISCUSSION}

\subsection{Characterization by an optical microscope:}

Parameter space test artefacts were inscribed within the bulk of fused silica, borosilicate and PMMA using the low-repetition Ti:Sapphire laser (Low-Rep 800). Figure 7 shows microscope images of the structures produced in fused silica for varying energies between $12.5 \mathrm{~mW}$ and $155 \mathrm{~mW}$ (or $5-100 \%$ of the set energy) (Figure 7a), speed (Figure 7b) and depth (Figure 7c). Similar results were also observed for PMMA and borosilicate. The structure inscribed in PMMA produced a smooth line around 0.7 $\mathrm{mm} / \mathrm{s}$, whereas in other materials it produced a pearl chain like periodic structure. This could be attributed to the softness and low melting point of the PMMA. At higher speed structures are more periodic, but at lower speed, for the same energy, damage is observed rather than pure index change. Structures inscribed at higher speed shows undulation in the OCT B-scans images.
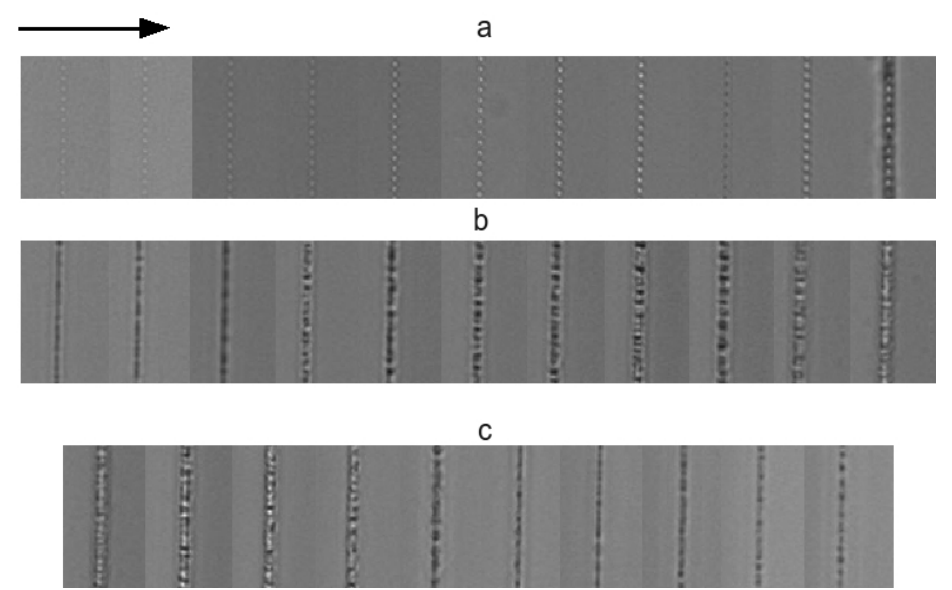

Figure 7: Microscope images parameter test artefact inscribed using Low-Rep 800 system, a) varying energy at $1 \mathrm{~mm} / \mathrm{s}, \mathrm{b}$ ) varying energy at $0.1 \mathrm{~mm} / \mathrm{s}$ inscribed at $100 \mathrm{um}$ below the front surface, c) effect of varying the depth from $100 \mathrm{um}$ to $675 \mathrm{um}$ for single energy and speed. 
Parameter space test artefacts were also inscribed within the bulk of fused silica and borosilicate glasses using the low-repetition Nd:YAG laser (Low-Rep1030). Microscope images of the structures produced in fused silica for varying energy, speed and depths are shown Figure 8. Figure 8a shows the variation of structure with increasing energy for a single depth and speed. The size of the modified region increases with increasing laser energy. Similar results were observed for borosilicate as well with structures slightly uniform than that is observed in the fused silica. All of the structures inscribed by Low-Rep1030 are either damage or micro voids.

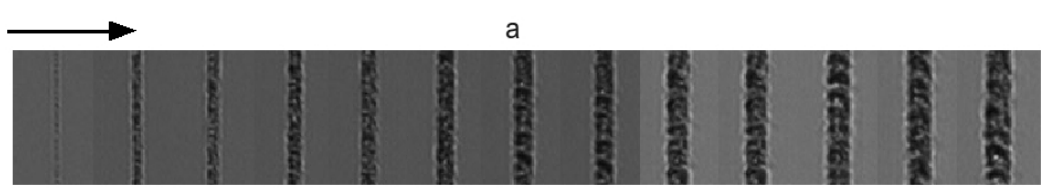

b

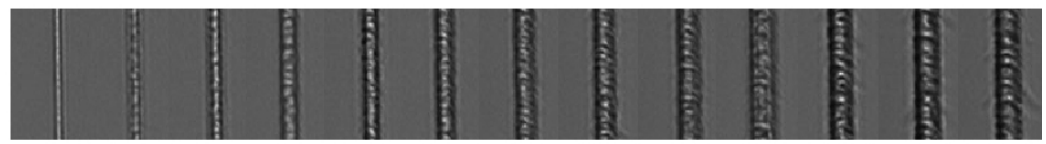

c

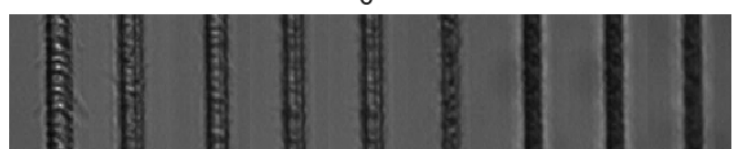

Figure 8: Microscope images parameter test artefact inscribed using Low-Rep1030 system, a) varying energy at $1 \mathrm{~mm} / \mathrm{s}$, b) varying energy at $15 \mathrm{~mm} / \mathrm{s}$ inscribed at $100 \mathrm{um}$ below the front surface, c) Effect of varying the depth from $100 \mathrm{um}$ to $525 \mathrm{um}$ for single energy and speed.

Figure 9 shows lines inscribed at varying energy by High-Rep800 system at constant speed and single depth. When this sample was cleaved and the cross sectional profiles analyzed using a microscope it could be seen that a circular modification was present. The reconstructed refractive index profile showed a waveguide like feature (i.e. high refractive index core surrounded by low refractive index cladding). The propagation losses for these waveguides at very low level (a few percent), thus indicating low absorption introduced by the HRR fs inscription ${ }^{16}$. It was the absence of imaginary part of permittivity which enabled to measure refractive indices by the QPM method ${ }^{17}$. This is unique feature and it is a consequence of heat accumulation regime ${ }^{18,19}$. Details of this work can be found elsewhere ${ }^{16}$.

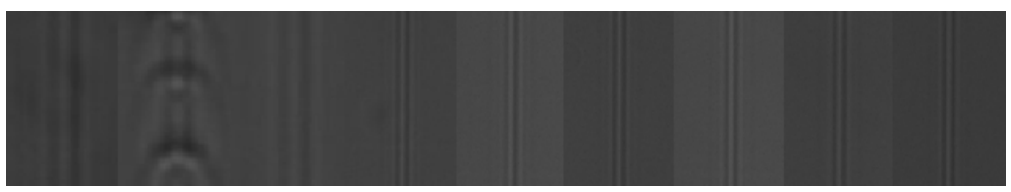

Figure 9: Typical microscope images of the structures inscribed at varying energy, constant speed and depth by $800 \mathrm{~nm}$ high-repetition system.

The phase of the inscribed structures was analyzed using a QPM technique. Figures 10a and b show the phase results from a typical lines inscribed by the Low-Rep1030 and High-Rep800 systems respectively. The phase induced by Low-Rep 800 changes from negative to positive with increasing speed due to different processes being (colour centres and densification ${ }^{21}$ )involve. 

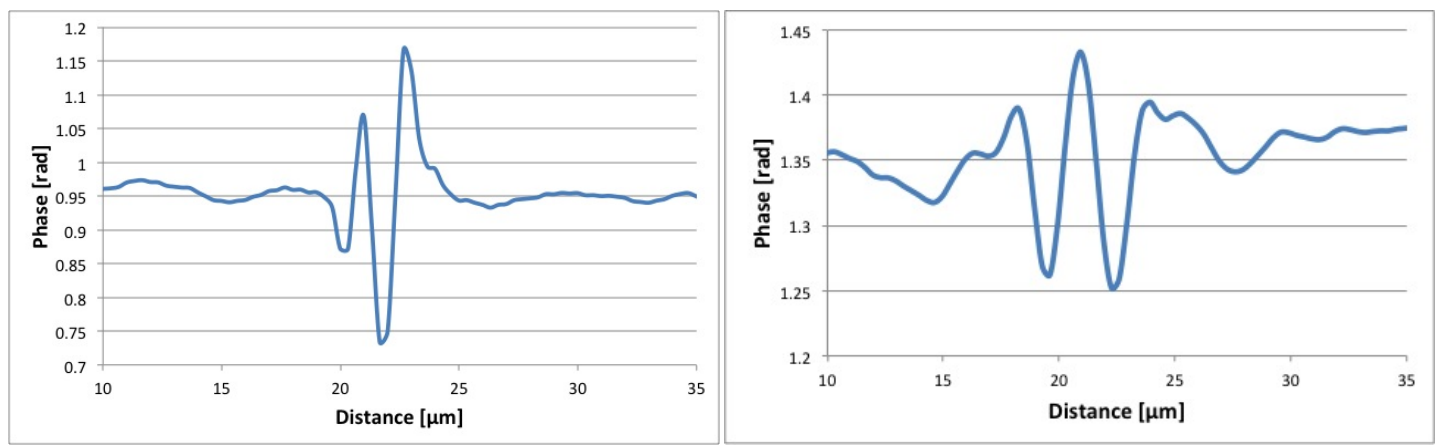

Figure 10: Phase measured using QPM for a typical structure inscribed by Low-Rep1030 (a) and High-Rep800 (b).

Figure 11 shows the variation of the inscribed structure with energy for a single speed and depth, measured from microscope images for Low-Rep1030 system. A more accurate structural size may be estimated from the phase measurement. The results show how the size of the structures increases with increasing energy. Similar trends were obtained from the other system and materials.

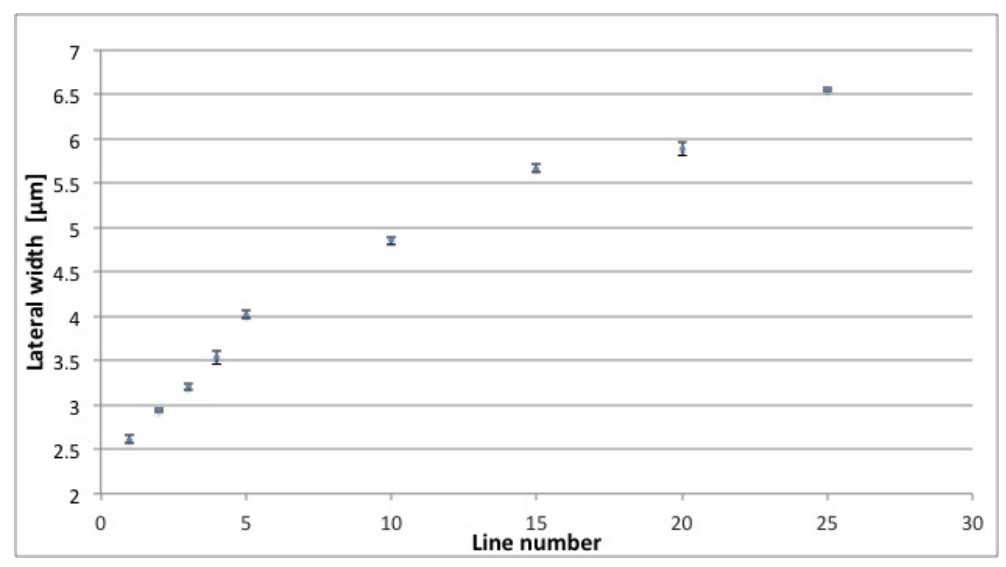

Figure 11: Variation of lateral size with increasing energy for constant speed and depth.

Figure 12 shows variation of the lateral size of the inscribed structures with increasing depth for constant speed and energy for Low-Rep1030 system. Results also indicate that the size of the structure decrease with increasing depth. For lower energies variation of the size with depth is relatively small compared to those structures written at higher energies.

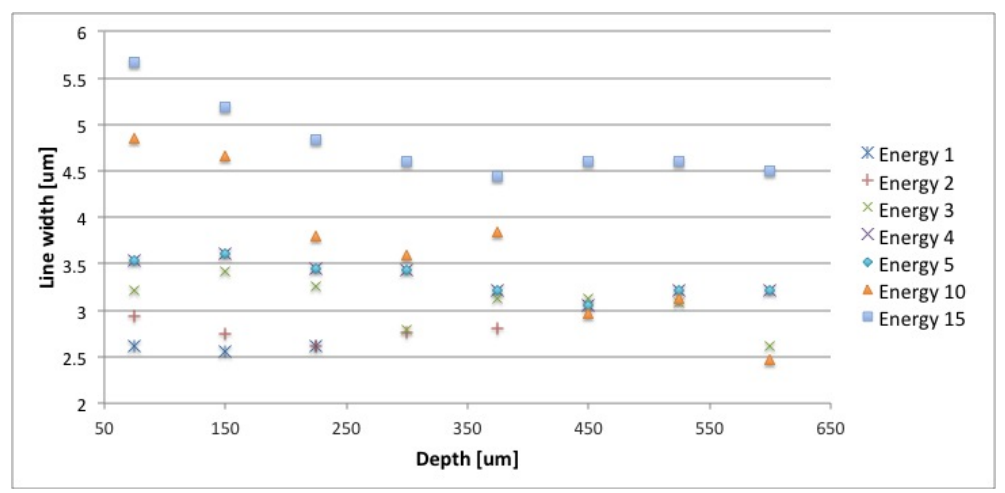

Figure 12: Variation of size with increasing depth for constant speed and energy. 


\subsection{Characterization using OCT}

The volumetric data was processed using an improved version of the CPU-GPU hybrid model described in [8], which achieves a processing rate of over 2.1 million A-scans per second. All the structures in the sample are at discrete locations this allows us to easily extract a subsection around each of the measurement points of interest. If less than six points within the selected subsection is with values smaller than the threshold criteria, then these were rejected. Only those subsections satisfying these conditions are selected for fitting.

Figure 13 shows the cross sectional profile of the structures (B-scan) of the first five lines pairs inscribed with the lowest energy inscribed in fused silica at $1 \mathrm{~mm} / \mathrm{s}$ translation speed for varying energy and depths. Lines within the pairs are identical, showing the reproducibility of the structures. Sub regions of the sample were over sampled and the dimensions of the structures were estimated from this. The line spacing, layer separation and layer offset are computed from the centroid data.

Furthermore, by calculating the centroid of each point in each frame, linearity of the lines can be estimated. Structures inscribed at lower energies, by both of the low repetition systems, look more symmetrical than those at higher energies (Figure $13 \mathrm{~b}, \mathrm{c}$ ). All of the structures inscribed using highrepetition system are nearly symmetrical (with cylindrical cross-sections).

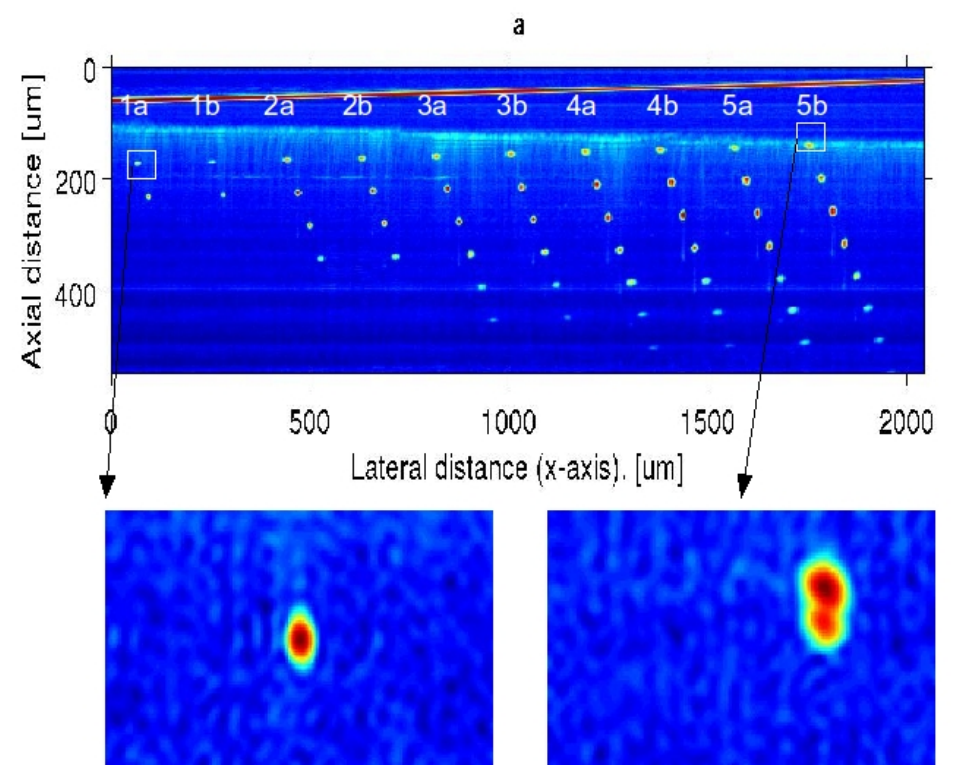

Figure 13: OCT B-scan of the first 5 pairs of lines from a sample inscribed by Low-Rep1030 system.

The reproducibility of the artefacts was verified by writing multiple lines and multiple artefacts with the same fabrication parameters. Figure 14 shows the OCT images of the first five pairs of the parameter space artefacts from Low-Rep1030 system. It is clear from the figure that the lines within the pairs are nearly almost identical, ensuring the reproducibility of the femtosecond inscription process. 


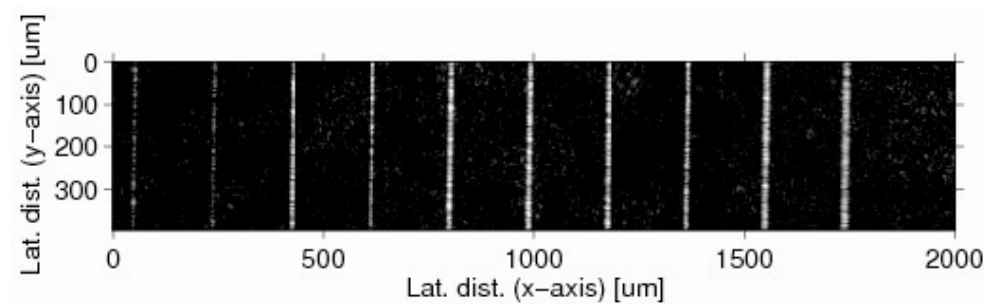

Figure 14: OCT image of five lines pairs inscribed by Low-Rep1030 systems.

\section{CONCLUSION}

In conclusion, this work compares the microstructures fabricated using three different femtosecond lasers with their suitability as optical coherence tomography artefacts in mind. The properties of the femtosecond inscribed structures from three different systems were analyzed. Structures fabricated within the fused silica, PMMA and doped-BK7 are observable by OCT, whereas those inscribed in borosilicate are not visible.

The results show that femtosecond inscribed structures are highly reproducible at certain fluences. Densification structures produced by the low repetition rate systems in fused silica and PMMA are more strongly visible in OCT measurements than the waveguide like structures produced by the high repetition system. Structures produced by the high repetition system are more symmetrical and homogeneous than those produced by low repetition systems. The size of elongation increases with increasing energy; however a cylindrical lens or slits can reduce this effect. Structures written with the Low-Rep800 system in PMMA tend to be more symmetrical than those in fused silica, perhaps because of the relaxation of the stress in the material.

Although, the induced refractive index changes of the structures inscribed in borosilicate using the High-Rep800 system were much larger than those in doped-BK7, and the samples from all three systems were clearly visible using the microscope, they were not visible with the OCT system..This is probably due to sample quality or material differences but this is still under investigation.

Waveguide like structure inscribed using high rate system (High-Rep800) are only visible to the OCT system within a limited depth, so this is ideal for fabricating structures with well-controlled structures with well-defined refractive index change (on a single layer) for sensitivity artefact but not good for characterizing depth. Structures inscribed with high fluence by Low-Rep systems are not as smooth as that of High-Rep systems, but those inscribed at lower fluence produce well-defined periodic structures. All system shows high reproducibility of the structures.

In conclusion, properties of these structures can be well characterized. The dimensions and positions of, as well as induced refractive index in the femtosecond inscribed microstructures can be well controlled by controlling the inscription parameters. Therefore it is realistic to produce standardization artefacts in this way with designs suitable for calibrating and characterizing OCT and other imaging systems.

\section{ACKNOWLEDGEMENTS}

We would like to acknowledge the assistance of Mykhaylo Dubov and Vladimir K Mezentsev from Aston University in providing the OCT artifact, inscribed using High-Rep800 system, used in this study and the financial support of NPL via a CASE studentship.

\section{REFERENCES}


[1] Huang, D., Swanson, E., Lin, C.P., Schuman, J.S., Stinson, W.G., Chung, W.,Hee, M.R.,Flotte, T., Gregory, K.,PuliafitoC.A. and Fujimoto, J.G., "Optical coherence tomography," Sciences 254, 11781181 (1991).

[2]Fercher, A.F.,Hitzenberger, C.K., KampG.,and El-Zaiat S.Y., "Measurement of intraocular distances by backscattering spectral interferometry," Opt. Commun. 117(1-2), $43-48$ (1995).

[3] Pogue B.W., and Patterson, M.S., "Review of tissue simulating phantoms for optical coherence spectroscopy, imaging and dosimetry," Journal of Biomedical Optics 11(4), 041102 (2006).

[4] Agrawal, A.,Pfefer, T.J.,Gilani, N., and Drezek, R., "Three-dimensional characterization of optical coherence tomography point spread functions with a nanoparticle-embedded artefacts," Optics Letters 35(13), 2269-2271 (2010).

[5] Tomlins, P. H., Ferguson, R. A., Hart, C., and Woolliams, P. D., "Point-Spread Function artefacts for Optical Coherence Tomography," (National Physical Laboratory, ReportOP2, Teddington, England, 2009).

[6] Tomlins, P.H., Smith, G. N.,Woolliams, P.D., Rasakanthan, J. and Sugden, K., "Femtosecond laser micro-inscription of optical coherence tomography resolution test artifacts," Bio. Med. Opt. Exp. 2(5), 1319 (2011).

[7] Ortiz, S., Siedlecki, D., Remon L., and Marcos, S., "Optical coherence tomography for quantitative surface topography," Applied Optics 48(35), 6708-6715 (2009).

[8] Woolliams, P.D. andTomlins, P.H., "The modulation transfer function of an optical coherence tomography imaging system in turbid media," Phys. Med. Biol. 56(9), 2855-2871 (2011).

[9] Yang, W. (PhD Thesis) "Femtosecond laser writing in transparent materials" (2008).

[10] Baum, A., Scully, P.J., Perrie, W., Liu, D. and Lucarini, V., "Mechanisms of femtosecond laserinduced refractive index modification of poly (methyl methacrylate)," JOSA B 27(1), 107-11 (2010).

[11] Gattass R. R. and Mazur, E., "Femtosecond laser micromachining in transparent materials," Nat. Photonics 2(4), 219-225 (2008).

[12] Schaffer, C. B., Jamison, A. O. and Mazur, E., "Morphology of femtosecond laser-induced structural changes in bulk transparent materials," Appl. Phys. Lett. 84(9), 1441-1443 (2004).

[13] Dubov, M., Bennion, I., Nikogosyan, D. N., Bolger, P. and Zayats, A. V., "Point-by-point inscription of $250 \mathrm{~nm}$ period structure in bulk fused silica by tightly focused femtosecond UV pulses,"

J. Opt. A Pure Appl. Opt. 10(2), 025305 (2008).

[14] Wang, D. L., Li, C. D.,Luo, L., Yang, H. and Gong, Q. H., "Sub-diffraction-limit voids in bulk quartz induced by femtosecond laser pulses," Chin. Phys. Lett. 18(1), 65-67 (2001).

[15] Dubov, M.,Allsop, T. D. P.,Natarajan, S. R., Mezentsev, V. K. and Bennion, I., "Low-loss waveguides in borosilicate glass fabricated by high-repetition-rate femtosecond chirp-pulsed oscillator," Lasers and Electro-Optics 2009 and the European Quantum Electronics Conference. CLEO Europe - EQEC 2009 CLEO-Europe and EQEC 2009 Conference Digest, CLEO Europe and EQEC, 1 (2009).

[16] Allsop, T.D.P., Dubov, M., Mezentsev, V. and Bennion, I., "Inscription and characterization of waveguides written into borosilicate glass by a high-repetition-rate femtosecond laser at $800 \mathrm{~nm}$," Applied Optics, v.49 (10), 1938-1950 (2010).

[17] Barty, A., Nugent, K. A.,Paganin, D. and Roberts, A., "Quantitative optical phase microscopy," Optics Letters 23(11), 817-819 (1998).

[18] Ampem-Lassen, E., Huntington, S.,Dragomir, N., Nugent, K. and Roberts, A., "Refractive index profiling of axially symmetric optical fibers: a new technique," Optics Express 13(9), 3277-3282

(2005). 
[19] S. M. Eaton, M. L. Ng, J. Bonse, A. Mermillod-Blondin, H. Zhang, A. Rosenfeld, and P. R. Herman, "Low-loss waveguides fabricated in BK7 glass by high repetition rate femtosecond fiber laser," Appl. Opt. 47, 2098-2102 (2008).

[20] S. M. Eaton, H. Zhang, M. L. Ng, J. Li, W-J.Chen, S. Ho, and P. R. Herman, "Transition from thermal diffusion to heat accumulation in high repetition rate femtosecond laser writing of buried optical waveguides," Opt. Express 16, 9443-9458 (2008).

[21] Streltsov, A.M., and Borrelli, N.F., "Study of femtosecond-laser-written waveguides in glasses,"JOSA B 19(10), 2496-2504 (2002) 